\title{
Focal Compression of the Upper Left Thoracic Intersegmental Arteries as a Potential Cause of Spinal Cord Ischemia
}

\author{
P. Gailloud, A. Ponti, L. Gregg, C.A. Pardo, and J.H.D. Fasel
}

\begin{abstract}
BACKGROUND AND PURPOSE: This study was prompted by recurrent angiographic observations of focal stenoses involving the proximal segment of the left upper thoracic intersegmental arteries, a few centimeters distal to their origin. The purpose was to investigate the nature and prevalence of this anomaly.
\end{abstract}

MATERIALS AND METHODS: The existence of non-ostial thoracic intersegmental artery stenoses was evaluated in 50 angiograms; the contribution of stenosed branches to the anterior spinal artery was recorded. Angiograms performed in 3 patients with right-sided aortas were also reviewed. The topographic relationships of the upper thoracic intersegmental artery with surrounding structures were investigated in 3 cadavers.

RESULTS: Thirty-seven non-ostial stenoses were found in 26 patients (52\%), predominantly on the left side (92\%), between T3 and T8, most frequently at T4 and T5 (54\%). Severe lesions were observed in 10\% of cases. Patients with stenoses had fewer detectable anterior radiculomedullary arteries between T3 and T5 (35\% versus 54\%). Upper intersegmental artery stenoses, documented on the left side of all 3 specimens, appeared to be caused by the recurrent path of these intersegmental arteries related to the leftward position of the thoracic aorta, and by their course around reinforced paramedian longitudinal strands of the endothoracic fascia.

CONCLUSIONS: Upper thoracic intersegmental artery stenoses are frequent. They result from the leftward deviation of the descending aorta and the existence of a fixed point along the course of the intersegmental arteries related to the endothoracic fascia. Because contributors to the spinal vascularization often originate at similar levels, these stenoses may play a role in the susceptibility of the upper and midthoracic spinal cord to ischemia.

ABBREVIATIONS: ISA = intersegmental artery; RMA = radiculomedullary artery

$\mathrm{T}$ his study was prompted by the recurrent observation made during spinal DSA of focal narrowings of variable severity involving the proximal segment of the left upper thoracic intersegmental arteries (ISAs), a few centimeters distal to their point of origin from the aorta (Fig 1). These stenoses can be severe and may result in the occlusion of the involved ISAs, in a manner reminiscent of the stenoses related to the passage of lumbar ISAs through the pillar of the diaphragm. ${ }^{1,2}$ The purpose of our study,

Received August 5, 2013; accepted after revision October 4.

From the Division of Interventional Neuroradiology (P.G., A.P., L.G.) and Department of Neurology (C.A.P.), The Johns Hopkins Hospital, Baltimore, Maryland; and Division of Anatomy (A.P., J.H.D.F.), University of Geneva Medical Center, Geneva, Switzerland.

Paper previously presented in part at: Annual Meeting of the American Society of Neuroradiology, April 21-26, 2012; New York, New York.

Please address correspondence to Philippe Gailloud, MD, Division of Interventional Neuroradiology, The Johns Hopkins Hospital, Bloomberg 7218, 1800 E Orleans St, Baltimore, MD 21287; e-mail: phg@jhmi.edu

http://dx.doi.org/10.3174/ajnr.A3833 conducted on clinical angiographic material as well as anatomic specimens, was to establish the angiographic prevalence of these focal narrowings, and discuss their etiology and potential clinical implications.

\section{MATERIALS AND METHODS \\ Radiologic Data}

The clinical data reviewed for this investigation were collected in institutional review board-approved databases; additional permission was granted for publication. The data were collected prospectively and stored in a dedicated workstation. All the studies were performed in a single-center by use of a biplanar neuroangiography suite (Artis zee; Siemens, Erlangen, Germany). To be considered, a spinal angiogram had to include bilateral evaluation of the ISA between T3-T12 and be free of pathologies involving the region of interest (eg, large vascular tumors). Fifty of a total of 132 spinal studies satisfying these criteria were performed between July 2011 and March 2013 in 21 women and 29 men, with age ranging from 3-80 years (average, 44; median, 47). Indica- 


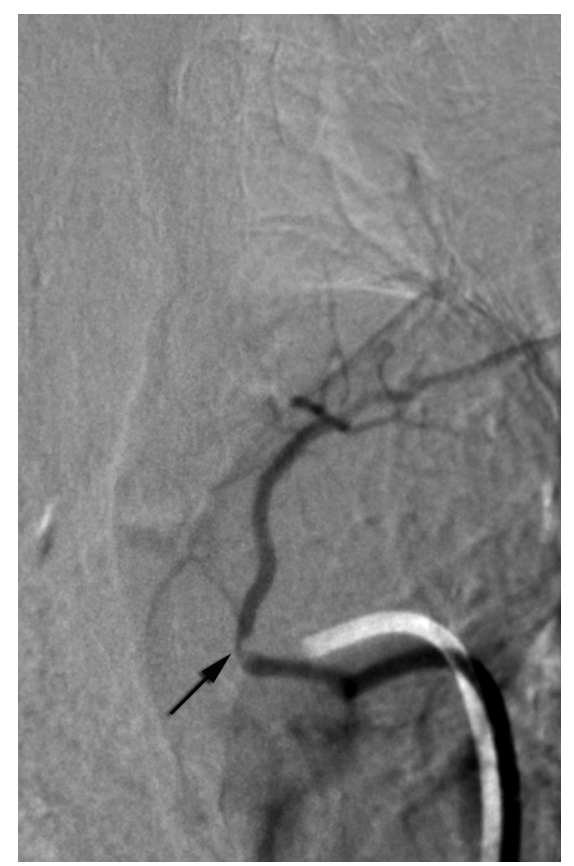

FIG 1. Non-ostial proximal intersegmental artery stenosis at left T4 in a 60-year-old woman (arrow).

tions for angiography in these 50 cases included progressive myelopathy (58\%), acute myelopathy (26\%), a known vascular malformation $(6 \%)$, and spinal cord hemorrhage or tumor $(10 \%)$.

Patients were logged in a normal group or a stenosis group on the basis of the presence of proximal narrowings, which were either categorized as 1) mild/moderate stenoses or 2) severe stenoses (including occlusions) (Fig $2 A-D$ ). Images were analyzed by use of a commercially available DICOM software (OsiriX 64, http://www.osirix-viewer.com), both in subtracted and native modes to assess for the presence of osseous changes potentially associated with arterial anomalies, for example, vertebral osteophytes. Available noninvasive imaging (CT and/or MR imaging) obtained in the patients with a stenosis was also reviewed.

The prevalence of upper thoracic radiculomedullary arteries (RMAs) has previously been analyzed in the same patient cohort and reported separately. However, radiculomedullary contributions coming from ISAs with a proximal stenosis were recorded, as well as the total number of anterior RMAs found between T3-T8 in both patient groups.

In addition, the presence of proximal stenoses was evaluated in 3 spinal angiograms performed by 1 of the authors in patients with right-sided aortic arches.

\section{Anatomic Data}

The topographic relationships of the upper thoracic ISA with surrounding structures were investigated in 3 cadavers.

\section{RESULTS}

\section{Radiologic Data}

Complete bilateral documentation of the ISAs between T3-T12 was achieved in 41 of the 50 reviewed angiograms (82\%). One upper thoracic ISA was not identified in 8 instances (left T3 in 6 cases, right $\mathrm{T} 3$ and right $\mathrm{T} 4$ once each) and 2 in 1 instance (left T3
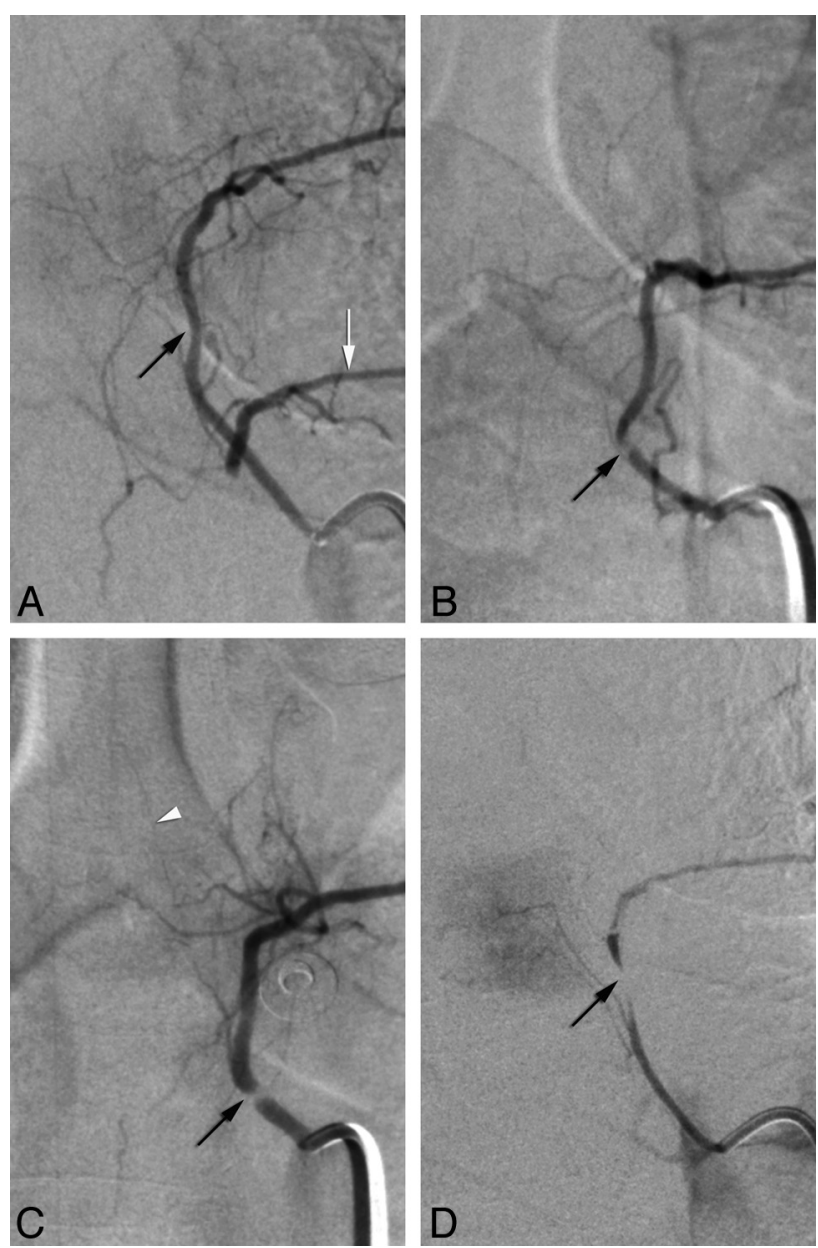

FIG 2. Categorization of stenoses as mild/moderate $(A$ and $B$ ) or severe ( $C$ and $D$ ) (from studied cohort). A, Mild stenosis at left T5 in a 69-year-old woman (black arrow) illustrates the mildest form of recorded narrowing. Note collateral flow to left T6 (white arrow), which harbors a near-occlusive lesion. $B$, Moderate stenosis at left T5 in a 41-year-old woman (arrow); a continuous column of contrast is seen across the narrowing. $C$, Severe stenosis at left T5 in a 40-yearold woman (arrow); although close in severity to the case shown in $B$, this narrowing shows a completely unopacified segment. White arrowhead points to an anterior radiculomedullary artery. $D$, Near-occlusion at left T6 in the patient shown in A (arrow).

and T4). Angiographic diagnoses included ischemic lesion (eg, diaphragmatic crus syndrome, spinal artery thrombosis) (28\%), a vascular malformation (14\%), and spine or spinal cord tumor (8\%); $50 \%$ of the angiograms were unremarkable.

Thirty-seven proximal non-ostial stenoses were noted in 26 of the 50 patients reviewed (52\%); 34 stenoses (92\%) were found on the left side and $3(8 \%)$ on the right. The level of origin of the stenosed ISAs ranged between T3-T8, most commonly T4 and T5 (54\%). Twenty-nine stenoses were of a mild/moderate degree (78\%), whereas 8 were severe $(22 \%)$; these severe stenoses were documented in 5 patients ( $10 \%$ of all patients), all on the left side. The Table summarizes our findings. The demographic characteristics of the 2 groups of patients were comparable: the 26 patients with stenoses included 12 women and 14 men, with an average age of 45 years (range, 3-80; median, 44), whereas the patients without stenoses included 9 women and 15 men, with an average age of 43 years (range, $8-66$; median, 48 ).

AJNR Am J Neuroradiol 35:1226-31 Jun 2014 www.ajnr.org 
Angiographic severity of proximal (non-ostial) stenoses affecting the upper thoracic intersegmental arteries

\begin{tabular}{cccccc} 
& \multicolumn{2}{c}{ Left } & & \multicolumn{2}{c}{ Right } \\
\cline { 2 - 3 } \cline { 5 - 6 } Levels & $\begin{array}{c}\text { Mild/ } \\
\text { Moderate }\end{array}$ & $\begin{array}{c}\text { Severe/ } \\
\text { Occlusions }\end{array}$ & & $\begin{array}{c}\text { Mild/ } \\
\text { Moderate }\end{array}$ & $\begin{array}{c}\text { Severe/ } \\
\text { Occlusions }\end{array}$ \\
\hline T3 & 3 & 1 & & \\
T4 & 8 & 1 & & \\
T5 & 7 & 3 & & \\
T6 & 3 & 2 & & \\
T7 & 1 & 1 & & & \\
T8 & 4 & & & & \\
\hline
\end{tabular}

No evidence of osseous anomalies (eg, osteophytes) was noted in the review of nonsubtracted angiographic images. The analysis of noninvasive studies (CT or MR imaging) in all 26 patients with stenoses showed no associated lesion and/or mass effect at the involved levels.

A stenosed ISA provided an anterior RMA in 5 instances ( mild $/$ moderate $=3$, severe $=2$ ) and a posterior RMA in 2 cases $($ mild $/$ moderate $=1$, severe $=1)$.

Sixty-six anterior RMAs were found between T3 and T8, with at least 1 anterior RMA found in 47 of the 50 patients (94\%). Thirty-two anterior RMAs were documented in the group without stenoses, with 23 of the 24 patients having at least 1 RMA (96\%). In the stenosis group, 34 anterior RMAs were seen, with 23 patients having at least 1 RMA (92\%). When considering only the branches found between $\mathrm{T} 3$ and $\mathrm{T} 5$, the number of patients with at least 1 anterior RMA was 9 in the stenosis group (35\%) and 13 in the normal group (54\%).

Proximal stenoses were found on neither side of the 3 patients with right-sided descending aorta.

\section{Anatomic Data}

Macroscopic investigations performed in 3 human specimens showed that the upper left thoracic ISAs initially adopt a medial course before sharply bending laterally and posteriorly in the vicinity of the vertebral column to pass along the posterior thoracic wall. This recurrent course and proximal curve was observed in all 3 specimens but not below the seventh thoracic vertebra or on the right side. To appreciate the anomaly, the thoracic aorta had to be gently dextroversed; the proximal portion of the ISAs could then be observed passing under bandlike reinforcements of the endothoracic fascia, mostly oriented longitudinally, some in a paramedian position, close to the medial aspect of the aorta (Fig 3A). The proximal portion of the ISA was bared by gentle blunt dissection of the endothoracic fascia. On both sides, the fascia tightly placated the arteries over the lateral aspect of the vertebral column. On the right side, the ISAs had a smooth course over the vertebral body before passing under the longitudinal reinforcements of the endothoracic fascia without noticeable curve or impingement. On the left side, the ISAs had an initial medial course that brought them to the fascia reinforcements, around which they took a sharp turn before continuing laterally and posteriorly within the intercostal space. The initial medial angulation of the artery and the sharpness of the turn around the edge of the fascia were most significant at the highest thoracic levels, correlating with a more lateral position of the aorta. Once freed
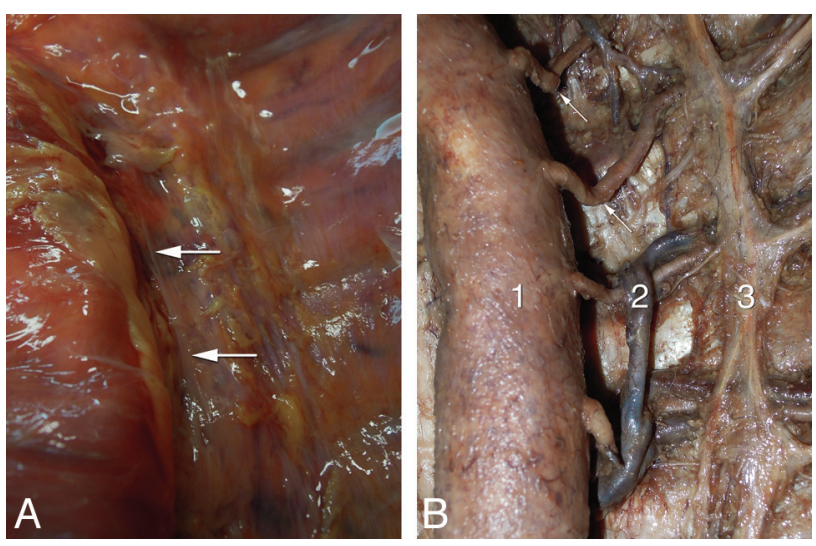

FIG 3. Anatomic observations in 2 human specimens. A, Anterior view of the endothoracic fascia in a fresh specimen after resection of the pleura; note the predominantly longitudinal bands of reinforcements (arrows) near the vertebral column. B, Anatomic dissection of an embalmed specimen documenting the proximal kinking of the left upper thoracic intersegmental arteries (arrows); 1, aorta (gently retracted to the right); 2 , accessory hemiazygos vein; 3 , sympathetic trunk.

from the attachment to the fascia, the proximal arterial segments showed a persistent bend, sometimes as severe as a kink (Fig 3B).

\section{DISCUSSION}

\section{Angiographic Findings}

The investigation of the spinal vasculature, first by nonselective and then by selective catheter spinal angiography, was pioneered in the mid-1960s by the teams of René Djindjian in France and John Doppman in the United States. ${ }^{3-6}$ Since then, the normal and pathologic angiographic appearance of the spine and spinal cord vessels has been extensively studied. ${ }^{5-13}$ Yet, a thorough review of the relevant anatomic and radiologic literatures, both past and current, did not yield specific information concerning the angiographic findings that prompted our investigation, for example, the observation of focal stenoses involving the proximal segment of the upper left thoracic ISAs. These non-ostial stenoses were found in $52 \%$ of our patients, mostly on the left side (92\%), without being influenced by age or sex. The most commonly involved levels were T4 and T5 (54\%). Eight of these stenoses (22\%) were severe, that is, preocclusive or occlusive; they were found in 5 of the 50 patients reviewed (10\%).

In a study examining the location of the aorta relative to the thoracic spine, Qiu et al ${ }^{14}$ observed the maximum deviation from the midline (in the normal control group) at T5, T6, and T7. This finding was confirmed by Cronin et $\mathrm{al},{ }^{15}$ who found the vertebralaortic angle (defined as the angle between the center of the vertebral body and the center of the aorta) to be at its highest value (approximately $239^{\circ}$ ) at T3, T4, T5, and T6, then progressively decreasing to reach its lowest value at the lumbar level (approximately $195^{\circ}$ ). The vertebral levels showing the highest deviation correspond to the sites of origin of the ISAs from T3-T7, ${ }^{16}$ levels at which were documented 33 of the 37 stenoses (89\%) seen in our patients. This correlation speaks strongly in favor of a relationship between the leftward deviation of the thoracic aorta and the prevalence of proximal ISA stenoses. The 3 mild right-sided stenoses found in our series showed a different pattern than the left-sided 


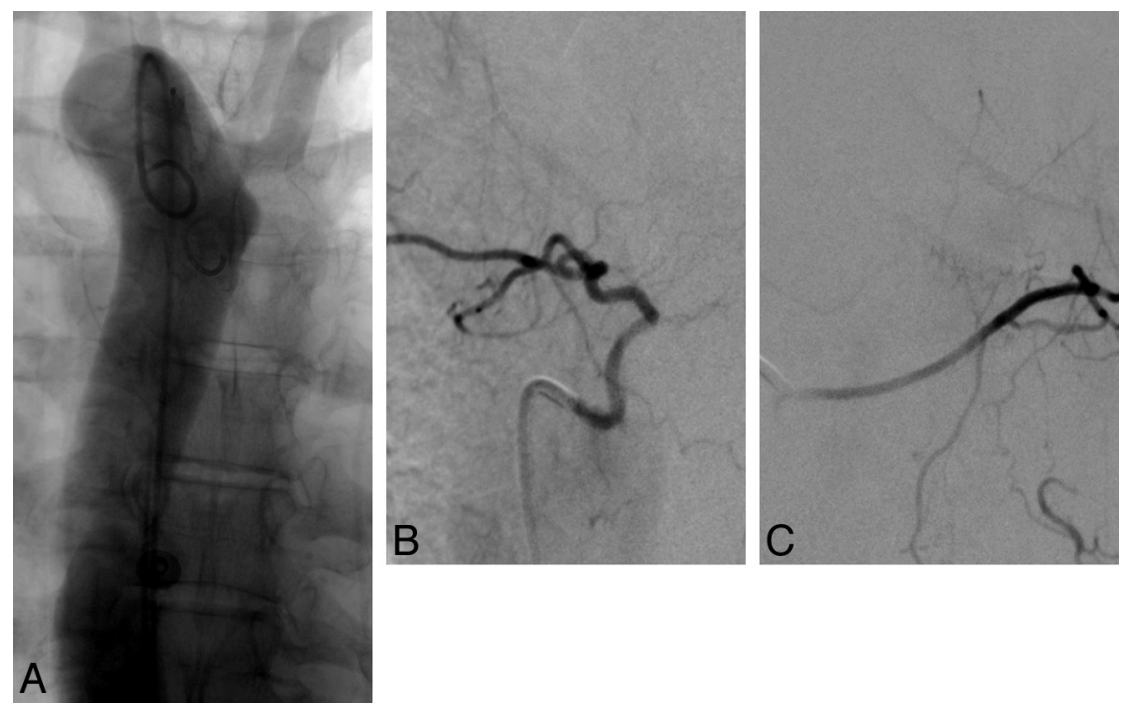

FIG 4. Mirror intersegmental anatomy in a 50-year-old woman with a right-sided aortic arch. A, Arch aortograms, postero-anterior projection; note that the deflection of the descending aorta from the midline is much less pronounced than in patients with a left-sided arch. B, Right T6 injection shows the typical course of a "normal" left-sided upper thoracic intersegmental artery (ISA), with a short recurrent course followed by a lateral bend. C, Left T6 injection shows the typical course of a "normal" right-sided upper thoracic ISA, with a smooth course across the midline, over the vertebral column.

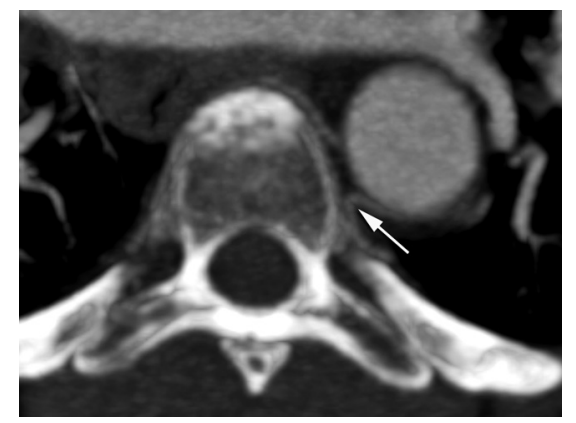

FIG 5. Thoracic CT angiography in a 54-year-old woman, axial image through the T5 level, illustrates the difference in trajectories between left and right upper thoracic intersegmental arteries, whereas the right-sided artery smoothly curves around the vertebral body to cross the midline; the left one, after a short initial recurrent segment, sharply bends dorsally to continue its course along the corresponding rib. This "fixed" point in the vessel path (arrow), which results from the obligatory turn the artery must take around the medial attachment of the endothoracic fascia, corresponds to the site of stenosis.

ones, that is, a short irregular segment rather than stenosis at a bend. We do not have a specific explanation for these three narrowings. On the other hand, it might be assumed that "bend" stenoses should be seen on the right side of patients with a rightsided aortic arch and descending aorta. None was found on either side in 3 such patients included in this report. This finding appears consistent with our hypothesis: the mirror anatomy of the upper thoracic ISAs explains the lack of stenoses on the left side, whereas the fact that right-sided thoracic aortas lie closer to the spine than left-sided ones renders them less likely to stretch rightsided ISAs and participate in stenosis formation (Fig 4).

Of note, in a few patients, selective angiography of an ISA harboring a proximal stenosis was performed during full inspiration and expiration; these maneuvers did not reveal a significant impact of the breathing pattern on the appearance and severity of the stenoses.

The low proportion of vascular malformations $(14 \%)$ included in the reviewed cohort of 50 cases (of a total of 132) probably reflects a selection bias, with patients with such a lesion being less likely to fulfill the inclusion criterion of having complete bilateral documentation of their thoracic ISAs.

\section{Anatomic Findings}

We initially postulated that upper thoracic ISA stenoses were caused by an extrinsic compressive mechanism, for example, by the sympathetic chain running alongside the vertebral column. Our anatomic investigations disproved this hypothesis because the sympathetic chain was too lateral to account for the stenoses observed angiographically. In addition, at the point in which the structures cross each other, the ISAs already lie in-between the ribs and appear well protected from potential compression by the sympathetic chain. On the other hand, focal bends of the upper left ISAs were seen in all 3 specimens investigated when the vessels were followed medially, near their site of origin from the dorsal aortic wall. The bends, visible only after gentle dextroversion of the aorta, resulted from the initial recurrent course of the upper thoracic ISAs and the sharp turn they assume around the medial reinforcements of the endothoracic fascia before continuing their course along the posterior thoracic wall. The severity of this bend was accentuated by the leftward position of the aorta at the upper thoracic level. The endothoracic fascia, reinforced by longitudinal strands of connective tissue, was found to act as a fixed point in the course of the ISAs, which were stretched by the lateral displacement of the aorta (Figs 5 and 6). The topography and appearance of the curves observed in the anatomic specimens correlated with most stenoses documented by means of spinal DSA in our patients.

\section{Clinical Implication}

Severe ISA stenoses were found in $10 \%$ of our patients, with anterior or posterior RMAs coming from these branches in 3 instances. Although our study is not large enough to provide meaningful statistical data, the fact that anterior RMAs were less often documented between $\mathrm{T} 3$ and $\mathrm{T} 5$ in the group of patients with stenoses ( $35 \%$ versus $54 \%$ ) is also interesting because it may suggest a relationship between proximal ISA lesions and impairment of the spinal cord blood supply. The lower angiographic prevalence of anterior RMAs in the stenosis group could be related either to suboptimal opacification or to thrombo-occlusive complications.

The upper and midthoracic regions of the spinal cord have been considered as a zone at risk for ischemia. Postulating a lack of adequate blood supply to that area, several authors introduced the concept of a thoracic arterial "watershed zone," 17,18 believed to 


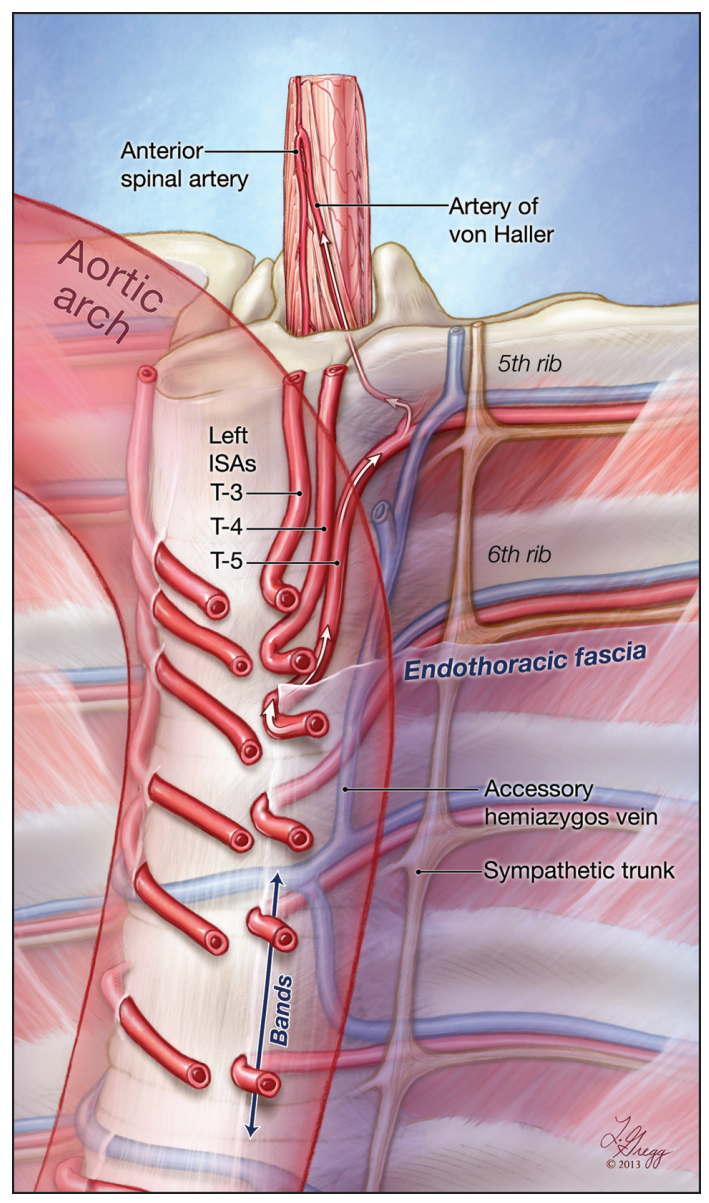

FIG 6. Graphic representation of the study findings. This illustration depicts the course of the upper thoracic intersegmental arteries (ISAs) in relation to the vertebral column, the endothoracic fascia, and the position of the thoracic aorta. Note the difference in trajectory between the right-sided ISAs, smoothly curving around the spine to pass under the fascia, and the left-sided ones, which have to adopt a recurrent course to reach the fixed paramedian points imposed by the attachment of the fascia to the lateral aspect of the vertebral column. The reinforced bands of connective tissue found within the paramedian endothoracic fascia are indicated by an arrow at $\mathrm{T} 7$ and T8. Note that the length of the recurrent segments and the sharpness of the bends increase with the leftward deviation of the upper thoracic aorta. In this example, the fifth thoracic ISA provides a prominent anterior radiculomedullary branch, the artery of von Haller.

result either from the transition of the spinal cord supply from the subclavian artery to the aorta, ${ }^{17}$ or from the smaller number and size of the thoracic anterior RMAs. ${ }^{19}$ Yet, this notion disregards the fact that if contributions to the spinal cord vascularization are indeed less conspicuous in the upper thoracic region than at the cervical or lumbosacral levels, the thoracic spinal cord is also of a lesser size, with a smaller volume of parenchyma to supply. Overall, as Gillilan ${ }^{20}$ observed, "the blood supply to the thoracic cord is entirely adequate for the volume of gray matter present, and it is relatively as good as for any other cord segment." Moreover, the presence of a constant anterior radiculomedullary artery at the upper or midthoracic level, playing a role similar to the arteries of the cervical and lumbosacral enlargements, has been suggested for some time..$^{21-24}$ In a recent angiographic study, this artery, for which the eponym of artery of von Haller was proposed, was found between left T3 and left T7 in approximately 70\% of
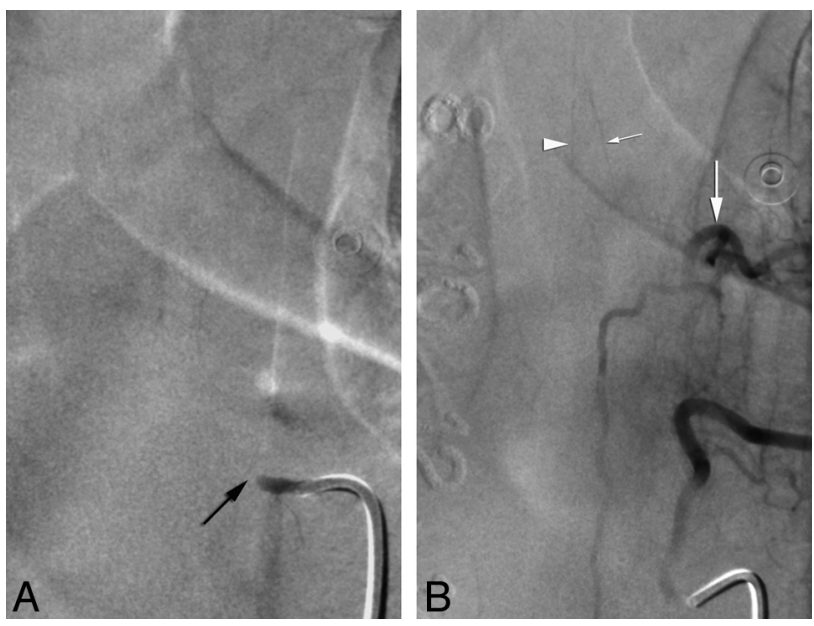

FIG 7. Angiographic findings investigating for progressive myelopathy in a 52-year-old woman. A, Non-ostial occlusion of the left T6 intersegmental artery. B, Left T7 injection shows collateral supply to left T6 distal to the site of occlusion (arrow), with opacification of the anterior spinal artery (arrowhead) through a left T6 anterior radiculomedullary artery (artery of von Haller, small arrow).

patients, weakening further the hypothesis that the thoracic spinal cord sensitivity to ischemia results from a "watershed" mechanism.

The findings reported in our study appear to provide an alternate explanation for the occurrence of upper and midthoracic spinal cord ischemia. The dominant anterior RMA at the upper thoracic level, or artery of von Haller, originates most commonly from the ISAs with the highest prevalence of proximal stenoses, that is, left T3-T7. The conjunction of these 2 anatomic conditions suggests the possibility that proximal stenoses impact the supply of the spinal cord by impairing the flow to the artery of von Haller (Fig 7). This mechanism would be very similar to the situation encountered at the lumbar level, in which the passage of upper lumbar ISAs through the crus of the diaphragm ${ }^{2}$ occasionally results in extrinsic compression, leading to spinal cord ischemia when the involved vessel provides a significant spinal artery contributor. ${ }^{1,25}$ Although surgical treatment can be offered at the lumbar level, with the compression being relieved by section of the pillar of the diaphragm, there is at this time no straightforward method addressing the upper thoracic ISA stenoses described in the present report. Several patients presenting with progressive or recurrent myelopathies of unclear etiologies, and in whom a stenosis or occlusion was shown to involve an ISA providing a prominent anterior RMA, were placed on prophylactic antiplatelet therapy with aspirin and/or clopidogrel.

\section{CONCLUSIONS}

Proximal non-ostial ISA stenoses were found at the upper thoracic level in approximately half the reviewed angiograms, in particular between T3-T8, almost exclusively on the left side. Severe stenoses or occlusions were observed in $10 \%$ of the patients investigated. These stenoses appear to result from the combination of the leftward deviation of the proximal descending aorta and the existence of a fixed paramedian point along the course of the ISAs, related to the attachment of the endothoracic fascia over the lateral aspect of the vertebral column. Because an important con- 
tributor to the spinal cord vascularization commonly originates from the ISAs with the highest prevalence of proximal stenoses (left T4 and T5), we suggest a potential role of this anatomic configuration in the susceptibility of the upper and midthoracic spinal cord regions to ischemia.

Disclosures: Philippe Gailloud_UNRELATED: Consultancy: Codman Neurovascular; Grants/Grants Pending: Siemens Medical; Patents (planned, pending or issued): Artventive Medical; Stock/Stock Options: Artventive Medical. Carlos A. PardoUNRELATED: Grants/Grants Pending: Bart McLean Fund for Neuroimmunology Research* ("money paid to institution).

\section{REFERENCES}

1. Rogopoulos A, Benchimol D, Paquis P, et al. Lumbar artery compression by the diaphragmatic crus: a new etiology for spinal cord ischemia. Ann Neurol 2000;48:261-64

2. Kauppila LI. Blood supply of the lower thoracic and lumbosacral regions: postmortem aortography in $\mathbf{3 8}$ young adults. Acta Radiologica 1994;35:541-44

3. Djindjian R, Faure C. [Neuro-radiological investigations (arteriography and phlebography) in vascular malformations of the spinal cord]. Roentgen Eur Radiodiagnostic Clinique Européen 1963;158: 171-95

4. Djindjian R, Houdart R, Hurth M. Acquisitions récentes en angiographie médullaire. Revure Neurologique 1966;115:1068-69

5. Doppman JL, Di Chiro G, Ommaya AK. Selective Arteriography of the Spinal Cord. St Louis: Green; 1969

6. Di Chiro G, Doppman J, Ommaya AK. Selective arteriography of arteriovenous aneurysms of spinal cord. Radiology 1967;88:1065-77

7. Djindjian R. Angiography of the Spinal Cord. Baltimore: University Park Press; 1970

8. Lazorthes G, Gouaze A, Djindjian R. Vascularisation et Circulation de la Moelle Épinière. Paris: Masson \& Cie; 1973

9. Launay M, Chiras J, Bories J. Angiography of the spinal cord: venous phase: normal features: pathological application. J Neuroradiol 1979;6:287-315

10. Di Chiro G, Wener L. Angiography of the spinal cord: a review of contemporary techniques and applications. J Neurosurg 1973;39: $1-29$
11. Merland JJ, Riche MC, Chiras J. Intraspinal extramedullary arteriovenous fistulae draining into the medullary veins. J Neuroradiol 1980;7:271-320

12. Chiras J, Morvan G, Merland JJ. The angiographic appearances of the normal intercostal and lumbar arteries: analysis and the anatomic correlation of the lateral branches. J Neuroradiol 1979; 6:169-96

13. Chiras J, Morvan G, Merland JJ. Blood supply to the thoracic (dorsal) and lumbar spine. Anat Clin 1982;4:23-31

14. Qiu Y, He YX, Wang B, et al. The anatomical relationship between the aorta and the thoracic vertebral bodies and its importance in the placement of the screw in thoracoscopic correction of scoliosis. Eur Spine J 2007;16:1367-72

15. Cronin P, Williams DM, Vellody R, et al. The normal distribution of thoracoabdominal aorta small branch artery ostia. Eur J Radiol 2011;80:e563-70

16. Shimizu S, Tanaka R, Kan S, et al. Origins of the segmental arteries in the aorta: an anatomic study for selective catheterization with spinal arteriography. AJNR Am J Neuroradiol 2005;26:922-28

17. Mettler FA. Neuroanatomy. 2nd ed. St Louis: CV Mosby Co; 1948

18. Zülch KJ. Mangeldurchblutung als Ursache ungeklärter Rückenmarksschädigungen. Deutsche Zeitschrift Nervenheilkunde 1954;172: 81-101

19. Dommisse GF. The Arteries and Veins of the Human Spinal Cord From Birth. Edinburgh/New York: Churchill Livingstone; 1975

20. Gillilan LA. The arterial blood supply of the human spinal cord. J Comp Neurol 1958;110:75-103

21. Von Haller A. Iconum anatomicarum quibus aliquae partes corporis humani delineatae traduntur. Fasciculus VII: Arteriae cerebri Medullae spinalis Oculi. Göttingen: Widow of Abram Vandenhoeck; 1754

22. Corbin JL. Anatomie et Pathologie Artérielles de la Moelle. Paris: Masson \& Cie; 1961

23. Suh T, Alexander L. Vascular system of the human spinal cord. Arch Neurol Psychiatry 1939;41:659-77

24. Djindjian R. Arteriography of the spinal cord. Am J Roentgenol Radium Ther Nucl Med 1969;107:461-78

25. Batt M, Rogopoulos A, Benchimol D, et al. Paraplegia with lumbar artery compression by the diaphragmatic crus. J Vasc Surg 2008;48: 1017-21 